\title{
In-situ Electrochemical Liquid Cell TEM Visualization of Electrode-Electrolyte Interfaces
}

\author{
Haimei Zheng ${ }^{1,2}$ \\ 1. Materials Sciences Division, Lawrence Berkeley National Laboratory, Berkeley, CA 94720 \\ 2. Department of Materials Science and Engineering, University of California, Berkeley, \\ CA 94720
}

We have developed electrochemical liquid cells for transmission electron microscopy (TEM), which allows imaging of electrochemical processes in liquid electrolytes with high spatial resolution in situ (Fig. 1). Using this set up, we study electrochemical deposition and dissolution of lead dendrites on gold electrodes immersed in an aqueous solution of lead nitrate. Observation reveals lead dendrites grow by the fast protrusion of lead branches in the electrolyte and tip splitting. A unique electrochemical growth of single crystal dendrites through nucleation, aggregation, alignment and attachment of randomly oriented small grains is identified [1].

Using the same development, we have also directly visualized electrochemical lithiation and delithiation of $\mathrm{Au}$ anodes in the commercial $\mathrm{LiPF}_{6} / \mathrm{EC} / \mathrm{DEC}$ electrolyte for lithium ion batteries [2]. We are able to monitor the changes of electrode-electrolyte interfaces while a cyclic voltammetry with the voltage range of $0 \sim-3 \mathrm{~V}$ and scan rate of $0.1 \mathrm{~V} / \mathrm{s}$ is applied between two electrodes. At the early stage, bubbles are formed by decomposition of electrolyte and subsequently lithiation of gold electrode followed by the growth of lithium dendrites is observed. The growth and evolution of solid electrolyte interface (SEI) thin film on another electrode are also captured, where there is an initial fast growth and the growth rate is dropped to almost zero when the voltage is decreased to $0 \mathrm{~V}$ (Fig. 2). The initial SEI film is in close contact with the Au electrode. Then a gap between the SEI film and the electrode starts to grow with the applied voltage. Additional work on controlling the SEI formation and electrolyte decomposition will be discussed. In situ observation of the electrode-electrolyte interfaces shed lights on strategies of improving electrode design for better performance and reducing short-circuit failure in lithium ion batteries.

\section{References}

[1] "Structural and Morphological Evolution of Lead Dendrites during electrochemical Migration", Sun, M., Liao, H., Niu, K., Zheng, H., Scientific Reports, 2013, 3, 3227.

[2] "Visualization of electrode-electrolyte interfaces in $\mathrm{LiPF}_{6} / \mathrm{EC} / \mathrm{DEC}$ electrolyte for lithium ion batteries via in-situ TEM.” Z. Zeng, W. Liang, H. G. Liao, H. L. Xin, Y.H. Chu, H. Zheng, Nano Lett. 2014, DOI: 10.1021/nl403922u.

[3] This work was supported by DOE Office of Science Early Career Research Program. 




Fig. 1. A scheme of in situ TEM experiments for the observation of electrochemical reactions using an electrochemical liquid cell. A cyclic voltammetry can be applied to the electrodes in the biasing liquid cell through a custom-made TEM holder.
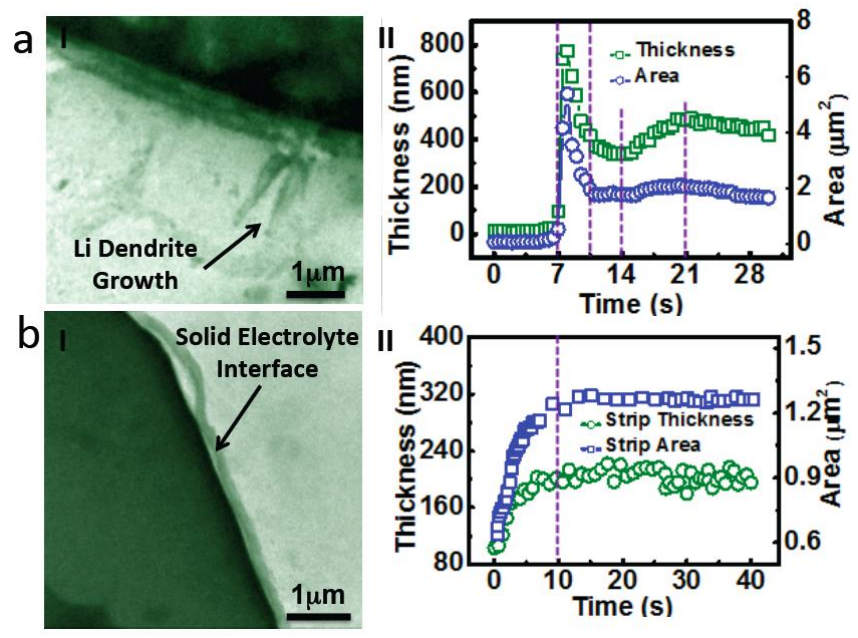

Fig. 2a The growth of a lithium dendrite (I) and the corresponding plot of Li-Au layer thickness and area as a function of time (II). b. Formation of SEI thin film (I) and the corresponding evolution of thickness and area of SEI layer with time (II). 